\section{Neuer Evidenzschub für die Hyposensibilisierung}

W

ird einem Patienten eine spezifische Immuntherapie (SIT) angeboten, sollten Sicherheit und Wirksamkeit des Präparats auf hohem Niveau belegt sein. Ein solcher Nachweis ist durch moderne randomisierte plazebokontrollierte Doppelblindstudien mit einem ausreichend großen Patientenkollektiv möglich und inzwischen auch Bedingung für die Zulassung von SITPräparaten. Eine Zulassung jüngeren Datums lässt also auf eine bestätigte Wirksamkeit schließen.

Doch wird von einem SIT-Präparat mehr verlangt, als nur die klinischen Symptome einer allergischen Rhinokonjunktivitis zu reduzieren. Ein wirksames Produkt sollte gemäß den EU-Richtlinien für SIT-Präparate auch einen über zwei oder drei Jahre anhaltenden Therapieerfolg sowie eine Langzeitwirkung nach Beendigung der Hyposensibilisierung bewirken, erläuterte PD Dr. Randolf Brehler, Münster. Wird dann langfristig auch noch ein „Heilerfolg“ im Sinne einer Normalisierung des pathologischen Immungeschehens nachgewiesen, kann das SIT-Präparat als echte kausale Allergietherapie empfohlen wer-

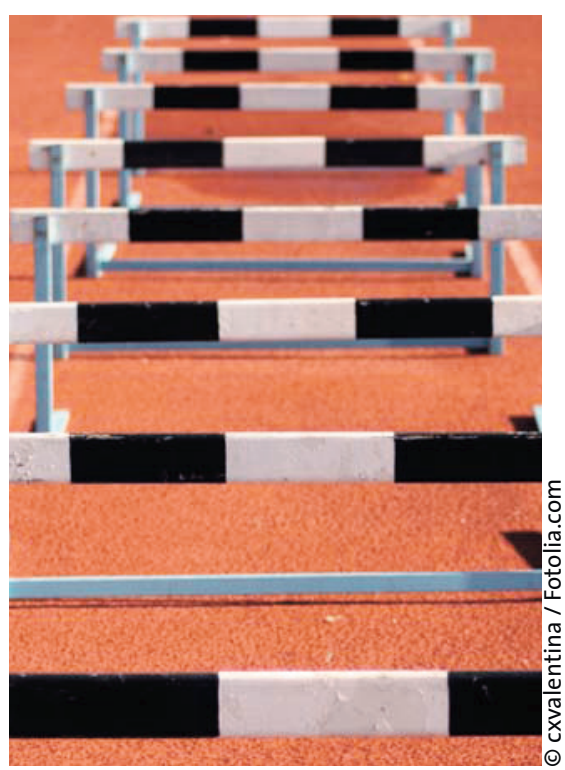

Bei der Zulassung von Präparaten zur spezifischen Immuntherapie sind heute deutlich mehr Hürden zu überwinden als früher. den. Aufgrund der fehlenden Vergleichbarkeit zwischen den verschiedenen Allergenpräparaten müssen die jeweiligen Nachweise für jedes Präparat gesondert erbracht werden.

Bei modernen SIT-Präparaten werden die genannten Anforderungen systematisch berücksichtigt und der Evidenzhorizont kontinuierlich ausgebaut. So bringt das Entwicklungsprogramm der Fünf-Gräser-Tablette Oralair ${ }^{\circledR}$ von Pollensaison zu Pollensaison neue Erkenntnisse. Zunächst standen hier die Suche nach der optimalen Dosis und die Frage nach der grundsätzlichen Wirksamkeit und Sicherheit im Vordergrund. Die positiven Studienresultate führten zur Zulassung von Oralair ${ }^{\circledR}$ für Erwachsene sowie auch für Kinder ab fünf Jahren. Dieses Jahr gehen die Studienpatienten nach drei aktiven Therapiezyklen nun erstmalig ohne die FünfGräser-Tablette in die Pollensaison. Ob sich in der aktiven Behandlungszeit ein guter Immunschutz aufgebaut hat und die Überlegenheit von Verum gegenüber Plazebo auch weiterhin anhält, wird die Auswertung der Patiententagebücher ab Herbst 2010 zeigen.

Noch in einer früheren Phase befindet sich das Stallergenes-Milbentabletten-Programm, berichtete Prof. Dr. Karl-Christian Bergmann, Berlin. Hier liegen erste Ergebnisse zur optimalen Dosierung und zur Wirksamkeit im ersten Behandlungsjahr vor, ermittelt unter Beteiligung von über 500 Patienten. Damit ist auch bei dieser wichtigen perennialen Allergie die Evidenz bei der SIT deutlich angehoben worden: Die Phase-III-Studie mit der Milbentablette ist die erste klinische Studie in der Milben-SIT überhaupt, in der die therapeutisch optimal wirksame Allergendosis ermittelt wurde. Die jetzt vorliegenden Daten sind die Basis für die angestrebte Zulassung der Milbentablette in Europa.

$b k$

Expert-Meeting „Die neue Dimension in der evidenzbasierten SIT“. Berlin, 20. Januar. Veranstalter: Stallergenes, KampLintfort

\section{Therapie mit Plan}

Die Durchführung einer sublingualen Immuntherapie erfordert einen individuell auf den Patienten abgestimmten Behandlungsplan, denn je nach Präparat und Allergen gibt es unterschiedliche Intervalle für die Kontrolltermine. Speziell für die Therapie mit der FünfGräser-Tablette Oralair ${ }^{\circledR}$ wurde deshalb der Oralair ${ }^{\circledR}$-Therapieplan entwickelt: Stellt man den Zeiger ,Termin 1 " auf den gewünschten Behandlungsbeginn, so ergeben sich auf einen Blick die drei nächsten Folgetermine. Darüber hinaus werden am äußeren Ring das Quartal sowie die Gräser- und Roggenpollensaison angezeigt. Auf diese Weise werden Arzt und Praxisteam bei der Vergabe der Folgetermine unterstützt. Für den Patienten wird der zeitliche Ablauf der Therapie optimal visualisiert.

Der Oralair ${ }^{\circledR}$-Therapieplan ist für Allergologen kostenlos erhältlich entweder über den Stallergenes-Außendienst oder telefonisch unter der Nummer (o 28 42) 90 40-1 46.

Nach Informationen von Stallergenes, Kamp-Lintfort

\section{Asthma unter Kontrolle?}

Die Therapie des Asthma bronchiale ist eine Herausforderung. Aktuelle Untersuchungen zufolge ist bei sieben von zehn deutschen Asthmapatienten die Krankheit nicht unter Kontrolle. Dabei können die Anzeichen für ein unzureichend eingestellten Asthmas durch gezielte Fragen schnell erkannt werden. Diese einfache Kontrolle unterstützt das Unternehmen Janssen-Cilag durch einen neuen Abreißblock, auf dem jeweils mit fünf Fragen der Kontrollstatus des Asthmapatienten eingeschätzt und gleichzeitig dokumentiert werden kann. Dieser Inuvair ${ }^{\circledR}$-Symptomblock ist kostenlos über den Außendienst des Unternehmens Janssen-Cilag erhältlich.

Nach Informationen von Janssen-Cilag, Neuss 